\title{
Una reflexión teórica al desarrollo de líneas de acción estratégicas orientadas al campesinado mexicano actual
}

\section{Diosey Lugo-Morin}

Especialista en Estrategias para el Desarrollo Agrícola Regional. Instituto Nacional de Tierras (INTi). Caracas. Venezuela

morin@colpos.mx / diosey@lycos.com

Resumen

El presente ensayo propone un conjunto de líneas de acción estratégicas orientadas a promover un desarrollo rural territorial centrado en el campesinado mexicano actual.

\section{Abstract}

The present article proposes a set of strategic attachment lines oriented to promote a rural development territorial trim in the present Mexican peasantry.

Palabras Clave: Desarrollo rural/ Desarrollo territorial/ Campesinado/ Organizaciones campesinas/ México

\section{I ntroducción}

México es hoy un país de grandes contrastes y de niveles de pobreza y déficit en indicadores sociales que están por arriba de lo esperado para un país con su grado de desarrollo. Tanto los índices de pobreza y desigualdad, como los indicadores sociales presentaron mejoras durante los años de crecimiento entre 1950 y 1980 . En los ochentas, durante la crisis de la deuda y su secuela, la pobreza y la desigualdad aumentaron, y los indicadores sociales si bien continuaron mejorando, lo hicieron a tasas menores. La leve y efímera recuperación económica de 1989 a 1992 resultó en una disminución de la pobreza, pero ésta todavía estaba por encima de los niveles de 1984. La tendencia no continuó entre 1992 y 1994 probablemente debido a la desaceleración de la tasa de crecimiento. Aunque todavía no se cuenta con información, la pobreza debe haber aumentado de manera muy marcada con la crisis del peso de 1995. (Lustig y Székely, 1997).

En 1992 se reformó el artículo 27 que otorgó a ejidos y comunidades la propiedad de la tierra, estableció normas para que las porciones de los socios pudieran circular. Suspendió las facultades jurisdiccionales extraordinarias otorgadas al poder ejecutivo en materia agraria. La intervención del gobierno en la economía rural se limitó a dar apoyos y subsidios frente a la competencia global. La desigualdad del campo mexicano en comparación a los países desarrollados y los sectores industriales y de servicios dentro del país es profunda. Más del $50 \%$ de la población se dedica a actividades agropecuarias, pero la mayoría no tiene los ingresos y bienes suficientes para vivir ni mejorar. 
A principios del siglo $X X, 30 \%$ del producto nacional provenía de actividades agrícolas, sin embargo esta riqueza se generaba bajo condiciones inequitativas, que condujeron a la revolución mexicana. Como resultado de ella, surge la constitución de 1917, que distribuye la tierra a sus antiguos dueños. El reparto agrario fue la acción pública más relevante de la primera mitad del siglo XX y partir de ella se legitima y alcanza consenso para abordar el modelo de urbanización e industrialización. Entre 1964 y 1970 se realizó el segundo reparto agrario tratando de resolver la crisis en la producción agropecuaria evidente a finales de 1960. Al mismo tiempo, el gobierno impulsó la formación de núcleos rurales competitivos y otorgó subsidios, sin resultados efectivos. A la par se dio el surgimiento de organizaciones campesinas que demandaban atención al campo, e inició una serie de confrontaciones entre éstas y las dependencias gubernamentales que no les daban solución (Warman, 2001).

Claramente, uno de los factores determinantes de la evolución de la pobreza ha sido el comportamiento macroeconómico. Desde la primera mitad de los setentas, México perdió la estabilidad que le caracterizaba y ha enfrentado crisis económicas recurrentes: en 1976, 1982, 1986 y 1994. En parte importante las crisis se explican por malas decisiones de política económica. Pero, también se explican por la vulnerabilidad de México al comportamiento de variables externas: los precios internacionales del petróleo en 1982 y en 1986, y, la tasa de interés externa y los flujos de capital en 1994. En el período reciente, la situación económica de México también se vio afectada por la mayor incertidumbre generada por la presencia de actos violentos y el proceso de transición a un sistema político más democrático y abierto (Lustig y Székely, 1997).

En este sentido, los cambios estructurales en el sector agropecuarios no se hicieron esperar. Ramírez y Peña (1999), destacan que a partir de la década de los 70's se observan signos de agotamiento del modelo de sustitución de importaciones. La agricultura pierde dinamismo y deja de generar recursos económicos para la industrialización. Esta enfrenta una estructura altamente polarizada, incapaz de seguir produciendo alimentos suficientes y baratos. La agricultura dejó de ser soporte de la industrialización y es marginada al no cumplir con la producción de alimentos y materias primas a bajo costo. En este marco se inició, a partir de mediados de la década de los 80, una profunda transformación del modelo de desarrollo bajo la política de ajuste estructural (Ávila, 1999). Para el sector agropecuario la política de ajuste estructural significó una profunda transformación de la estructura productiva como de la política agrícola. A partir de 1986 se inició la desregulación del comercio agroalimentario y la importación de grandes cantidades de alimentos. Se buscó en el exterior la obtención de alimentos y materia primas abundantes y baratas ante una agricultura en crisis.

Esto afectó a vastos sectores productivos, particularmente a los campesinos, al ocasionar la competencia mercantil con precios inferiores a los internos. En la etapa de globalización una agricultura atrasada productiva y tecnológicamente se convierte en un obstáculo para la acumulación de capital. En este contexto, el sector agropecuario ha sufrido una profunda transformación productiva. Los sectores más dinámicos se orientan hacia cultivos de mayor rentabilidad que demanda el mercado internacional y los estratos de altos ingresos del país en detrimento de la producción de granos básicos y oleaginosas (Ramírez y Peña, I bíd). 


\section{Perspectivas para el desarrollo de líneas de acción estratégicas}

Esta aproximación teórica sobre la problemática del campo mexicano, es factor importante a la hora de elaborar líneas de acción estratégicas orientadas a promover un desarrollo territorial rural donde el factor clave esta representado en la gran masa campesina que hace vida en el campo mexicano.

Elaborar líneas de acción estratégicas para solventar la realidad del campo mexicano actual requiere de algunos elementos, entre los que destacan: El territorio, el campesino, la coordinación institucional como eje integrador, el sector privado y el campesinado actual, la innovación tecnológica y el conocimiento local o indígena, todo enmarcado en un proceso relacional. Se destaca que la CEPAL, según lo señala Silva (2003), ha realizados esfuerzos importantes orientados a la construcción de herramientas metodologicas que sirvan de marco para proponer estrategias de desarrollo local y regional, sin embargo considero que algunas de las herramientas de análisis no son las más adecuadas, en términos de poderlas aplicar a ciertas realidades. Entre las debilidades marcadas del modelo de la CEPAL esta en que el campesino no es relevante a la hora de diseñar estrategias, aspecto que considero clave en este ensayo.

En su trabajo Silva (2003), señala un conjunto de fases con las técnicas asociadas a su formulación:

Diagnostico: Debe proporcionar la información que permite conocer la capacidad de desarrollo, las oportunidades y potencialidades, así como los recursos disponibles para ello. En el caso de este ejercicio, se propone arrancar desde los diagnósticos preexistentes de que dispone el respectivo gobierno local, los cuales están basados en información de base secundaria y que deben recoger los estudiantes para conformarse un panorama preliminar de la situación de la localidad. A estos antecedentes se sugiere agregarle información propia recolectada a través de dos mecanismos: entrevistas a informantes claves y observación directa con visitas a terreno. Recurriendo a estos antecedentes se propone entonces que los participantes de este trabajo reúnan los elementos preliminares necesarios para formarse una idea general de las potencialidades y obstáculos que la localidad presenta para activar un proceso de desarrollo local.

Vocaciones: Definidas como la aptitud, capacidad o característica especial que tiene la localidad para su desarrollo. En definitiva, se trata de buscar que es lo que hace especial, propio del lugar, como imagen de marca diferenciada a la localidad para potenciar algunas actividades estratégicas que le permitieran impulsar un proceso de desarrollo específico: ¿La ciudad o territorio, tiene vocación productiva, a partir de la explotación de algunos recursos propios? ¿Tiene vocación turística? ¿Reúne condiciones para ser un centro de servicios? Para la realización de esta parte del ejercicio, se sugiere, a partir de cada una de las áreas de trabajo que se han identificado aspectos económico-productivos; aspectos socioculturales; infraestructura y servicios públicos; aspectos institucionales - construir, con la ayuda de los antecedentes del diagnóstico, una matriz en la cual se anoten las potencialidades y limitaciones que se encuentran para cada uno de estos sectores, y del análisis de los mismos procurar definir cuales podrían ser las vocaciones de la localidad. En una tercera columna se 
Una reflexión teórica al desarrollo de líneas de acción estratégicas orientadas al campesinado mexicano actual

propone anotar los principales problemas que se detectan para el desarrollo de cada uno de ellos.

Objetivos estratégicos y específicos: A partir de los problemas enumerados en la fase anterior se deben, utilizando la técnica de análisis de árboles de problemas, identificar los árboles de medios y fines y la situación esperada que significaría la resolución de los problemas detectados. Los fines y la situación esperada asociados a esta fase se transformarían en los objetivos estratégicos y específicos que se deberían alcanzar, los mismos que se pueden definir como las aspiraciones en cada ámbito de desarrollo en que se ha dividido el ejercicio.

Estrategia local de desarrollo: La estrategia se define como el camino seleccionado para alcanzar los objetivos propuestos. Para ello se recurre a una técnica de análisis FODA de manera tal de estudiar para cada objetivo estratégico las variables internas (fortalezas y debilidades) y externas (oportunidades y amenazas) que pueden condicionar o viabilizar el alcance de los objetivos. Para ello, se debe procurar la definición de la estrategia más adecuada aprovechando al máximo las potencialidades (fortalezas y oportunidades), minimizando los riesgos (fortalezas y amenazas), enfrentado los desafíos (debilidades y amenazas), y procurando neutralizar las limitaciones (debilidades y amenazas).

Proyectos de inversión: Para materializar los objetivos de desarrollo, a través de la estrategia seleccionada, se requiere identificar y seleccionar los proyectos de inversión que darán concreción efectiva al plan de desarrollo. Los proyectos se pueden identificar a partir de los medios del árbol de medios y fines utilizado para definir los objetivos. Este ejercicio de planificación concluiría en este punto con la enumeración de algunas ideas de proyectos estratégicos cuyo desarrollo e implementación podría quedar como un desafío para las respectivas autoridades locales.

\section{El territorio}

Cuando nos referimos al territorio, no hablamos estrictamente de un espacio físico objetivamente existente, sino como una construcción social, es decir como un conjunto de relaciones sociales que dan origen y a la vez expresan una identidad y un sentido de propósito compartido por múltiples agentes públicos y privados (aunque dicha construcción implique muchas veces transitar por procesos de conflicto y negociación). Es dicha identidad la que permite dar sentido y contenido a un proyecto de desarrollo de un espacio determinado, a partir de la convergencia de intereses y voluntades.

En este sentido, partimos de La Ley de Desarrollo Rural Sustentable, promulgada en el 2002 y donde se adoptan numerosos elementos de enfoque territorial. En la ley, el territorio rural se conceptualiza como el ámbito construido a partir del uso y apropiación de recursos naturales, donde se generan procesos productivos, culturales, sociales y políticos, nacidos del efecto de localización. El enfoque territorial se adopta así como una condición para la integralidad de las políticas de desarrollo rural.

Berdegué y Schejtman (2003), señalan que este enfoque territorial se operacionaliza propiciando la programación del desarrollo rural sustentable de cada entidad federativa y de los municipios; promoviendo la formulación de programas a nivel municipal y regional o de cuenca, a través de los Distritos de Desarrollo Rural (DDR), e impulsando la federalización y la descentralización y permitiendo que a través de los Consejos 
Estatales, Regionales y Municipales se definan las prioridades regionales. Particular atención merecen los Distritos de Desarrollo Rural, los que de acuerdo con la ley deben ser la base: de la organización territorial y administrativas de las dependencias de la Administración Pública Federal y Descentralizada; para la realización de los programas operativos de la administración Pública Federal, y para la concertación con las organización de productores y los sectores sociales y privados. Los DDR son unidades de planificación y operación definidas a nivel central por la Secretaria de Agricultura, Ganadería, Desarrollo Rural, Pesca y Alimentación (SAGARPA), en consulta con los Consejos Estatales (provinciales) de Desarrollo Rural. En las regiones rurales con presencia significativa de población indígena, los DDR se delimitan considerando esta composición, con la finalidad de proteger y respectar los usos, costumbres y formas especificas de organización social indígena.

La Ley de Desarrollo Rural es el motor principal para potencial el desarrollo territorial rural de abajo-arriba, sin embargo existen dos elementos fundamentales que deben considerarse a la hora de su reglamentación, como lo es la importancia que revisten los sujetos sociales y la puesta en marcha de la propia ley en si (con que recursos económicos y financieros se cuenta y de donde saldrán los mismos).

\section{El campesino}

La conceptualización del campesino es un aspecto relevante, ya que cuando enfrentamos el análisis de la realidad socio-histórica, por lo general nos encontramos que los fenómenos sociales no sólo no encuadran dentro de los límites disciplinarios usuales, sino que además crece la incertidumbre con respecto a la posible objetividad del conocimiento. Siguiendo a Calvillo y Favela (1995) el planteamiento teórico acerca de la conceptualizacion del campesino toma una dimensión importante debido a que la crisis socio-económica esta transformando a la sociedad mexicana, donde están emergiendo nuevos sujetos sociales producto de las dinámicas productivas locales. En este sentido, los viejos esquemas que permitían una aproximación al entendimientos de dichos fenómenos, están obsoletos o invalidados, ya que no presentan una relación con la realidad que se esta gestando.

En un fin de siglo marcado por el productivismo, los campesinos no pueden quedarse al margen; la nueva socialidad rural ya no sólo tiene sus nudos en barrios y comunidades, también en proyectos y aparatos económicos gestionados por las organizaciones. Las empresas medianas o grandes de carácter asociativo son parte del nuevo perfil campesino y los conocimientos y las habilidades necesarias para operarlos se están incorporando a su cultura. Los campesinos no han dejado se ser los productores familiares de siempre, pero hoy son también gestores colectivos de empresas de servicios o agroindustriales; emplean añosos sistemas de cultivo, lo que no les impide coquetear con la más novedosa biotecnología; pueden cosechar maíz, fríjol y calabaza para el autoconsumo al tiempo que abastecen el mercado gourmet de Nueva York. El núcleo duro de la socialidad campesina esta aun en la comunidad agraria, pero su mundo ya no termina a orillas de la aldea (Bartra, 1998).

Una aproximación a la construcción conceptual del campesino se orienta a que es un ser que esta posicionado en un mundo fenomenológico social, él se ubica en el contexto histórico, ético, folklórico y religioso y difícilmente puede ser definible, ni siquiera indefinible, debido a la multiplicidad de roles que cumple en la sociedad 
Una reflexión teórica al desarrollo de líneas de acción estratégicas orientadas al campesinado mexicano actual

actual. El campesino actual es una mezcla de pasado, presente y futuro, esta afirmación en definitiva es la clave de su reproducción social (Lugo, 2005).

En el análisis del campesinado actual se puede constatar el carácter estratégico que juega la participación campesina en las políticas de desarrollo rural, su participación deviene en el potenciamiento del capital social como basamento de las relaciones sociales que a nivel comunitario constituyen redes de confianza, solidaridad y reciprocidad, originando un sistema reproductor del desarrollo económico local (Calvillo y Favela, 1995). Se destaca que todo esfuerzo orientado a promover o diseñar estrategias de desarrollo local debe estar centrado en el sujeto social ${ }^{1}$.

\section{La coordinación institucional como eje integrador}

La articulación institucional es un proceso complejo que debe darse, y que debido a las particularidades y objetivos que tiene cada dependencia oficial, ya sea de carácter federal o estatal, se ha retardo. Este aspecto tal como lo mencionaban Berdegué y Schejtman (2003), tiene rango constitucional (Articulo 27) y esta contemplado en la Ley de Desarrollo Rural Sustentable. Haciendo un análisis de los aspectos mencionados, es relevante en estos momentos una coordinación institucional en el ámbito del desarrollo rural que abarque los esfuerzos de la municipalidad y los planes de desarrollo que a bien estén en manos del DDR. Esta cooperación para el desarrollo rural local debe potenciarse en todos los niveles administrativos, la coordinación de las actuaciones agrícolas, pecuaria y ambiental, en el marco de la Ley de Desarrollo Rural Sustentable, facilitaría la implementación y ejecución de cualquier estrategia que se proponga. Otro aspecto relevante que potenciaría la coordinación institucional es la provisión de infraestructura y equipamiento rural. Para ello, las actuaciones deberán coordinarse a través de una comisión que pudiera ser de carácter temporal o permanente, dependiendo de los acuerdos a que lleguen, la misma podría estar integrada por un representante de la presidencia municipal, un del DDR, y uno por cada programa que hace vida en el municipio involucrado. Esta comisión se encargaría de darle seguimiento in situ a las estrategias propuestas, así como de elaborar planes y proyectos requeridos por las instituciones.

\section{El sector privado y el campesinado actual}

Actualmente la sociedad ha impuesto normas a todos los niveles de la esfera económica, social, política, ambiental y hasta cultural, bajo este contexto ha surgido un nuevo orden mundial, el cual ha hecho que las economías de los países periféricos reorientes sus políticas en función de poder posicionarse en estos escenarios. En este sentido, los gobiernos y sus políticas están orientados a competir bajos condiciones muy desiguales, es el caso por sólo dar un ejemplo de los subsidios en los países centrales, los cuales protegen de manera íntegra al agricultor. Caso que no sucede en los países periféricos donde los agricultores no poseen ningún tipo de subsidio en los términos que se dan en los países centrales ocasionando que pequeños y medianos agricultores no puedan competir y en consecuencia estos son absorbidos por los grandes agricultores, generando en los pequeños y medianos productores un cambio de estrategia de subsistencia, convirtiéndose en asalariados o más grave aún que los mismos vean como una alternativa la migración.

En este contexto donde existe una relación de poder entre los países centrales y los países periféricos, estos últimos han reformados su estructura legal en función de que 
desaparezca el pequeño agricultor o campesino. Se destaca que los pocos campesinos que quedan en estos países y que intentan organizarse son literalmente obligados ha operar bajos esquemas competitivos, sin considerar que estos sujetos sociales poseen una estructura valorativa distinta a la existente en una organización de tipo empresarial. Es importante destacar que desde los años 60's se trato de imponer el concepto de finca familiar norteamericana, este fenómeno se afianza y se convierte en el modelo de unidad productiva de una cierta corriente ideológica que busca la modernización de la agricultura a través de la reforma agraria y la colonización de nuevas tierras. Se le concibió como el tipo de empresa agraria más coherente con una acelerada tasa de adopción de los cambios técnicos en la agricultura. Se hace la anterior aseveración, debido a que muchos programas y proyectos de corte federal y Estatal tratan de imponer criterios de eficiencia empresarial a las organizaciones de campesinos, es probable que debido a estos lineamientos muchos de los programas y proyectos con orientación social y económica fracasen.

Cuando hablamos de organización campesina nos imaginamos a un grupo de personas reunidas en torno a un objetivo. Por otra parte, cuando hablamos de organización empresarial nos imaginamos un conjunto de relaciones, asignación de tareas, eficiencia, productividad. En cada tipo de organización existen marcadas diferencias, sin embargo siempre se trata de imponer el esquema de la organización empresarial a las organizaciones campesinas sin considerar que las relaciones entre los individuos se dan en condiciones distintas (Cuadro 1). En este aspecto, los sujetos han evolucionado en términos de organización donde la relación de poder ha prevalecido como forma de cumplir sus objetivos y obtener recursos para su sostenimiento.

Cuadro 1. Diferencias entre una organización campesina y una organización empresarial

\begin{tabular}{c|c|c|c}
\hline \multicolumn{1}{c|}{ Organización empresarial } & \multicolumn{1}{c}{ Organización campesina } \\
\hline $\begin{array}{l}\text { La organización dispone de recursos para } \\
\text { operar. }\end{array}$ & $\begin{array}{l}\text { Requieren del componente político para la } \\
\text { consecución de los recursos y subsidios. }\end{array}$ \\
$\begin{array}{l}\text { La organización opera en función de un } \\
\text { sistema administrativo. }\end{array}$ & $\begin{array}{l}\text { La organización opera en función del } \\
\text { conocimiento tácito de sus miembros. }\end{array}$ \\
\hline Existe una jerarquización pre-establecida & $\begin{array}{l}\text { Existe una jerarquización basada en } \\
\text { iniciativas }\end{array}$ \\
\hline
\end{tabular}

Es por esta razón, que a la hora de formular programas de desarrollo orientado a la metátesis de las organizaciones campesinas a organizaciones empresariales con criterios de competitividad se debe considerar elementos como la estructura de valores, necesidades, identidad colectiva, conocimientos locales, etc., las organizaciones en primera instancia operan como asociación los socios unen esfuerzo en función de un objetivo, una vez consolidados comienzan a operan con criterios administrativos y se asignan tareas para la consecución económica y posterior reproducción de la estructura organizativa.

La relevancia que le dan, Berdegué y Schejtman a los Distritos de Desarrollo Rural (DDR) como eje articulador para impulsar el desarrollo económico local, es punto que considero de importancia, pues pongo en la palestra el caso de la comunidad de San Luís Sesma, ubicada en el municipio de Mazapiltepec del Estado de Puebla. La 
comunidad de San Luís Sesma tiene una población de 197 habitantes, están organizados por unidades familiares campesinas. San Luís Sesma, esta marcada por una nueva socialidad rural construida en base a necesidades emergentes y nuevas formas de vidas, es decir, se han agrupados para la adquisición de maquinaria y equipos agrícolas, mercado (venta de productos como leche), los jóvenes residentes han sentido la necesidades de abrirse a nuevos espacios impulsados por un lado por la falta de empleo y por el otro lado impulsados por las expectativas del mundo que rodea a su comunidad generando de manera incipiente el fenómeno de la desterritorización. El campesino de San Luís Sesma en términos económicos es un trabajador rural por cuenta propia con acceso a la tierra, pero esto no significa que tengan equilibrio productivo y autosuficiencia. El núcleo de la socialidad campesina en San Luís Sesma esta aun en la comunidad agraria, pero su mundo ya no termina en los limites de la localidad.

Considerando este enfoque, la localización de las actividades agrícolas toma simultáneamente en cuenta el punto de vista agronómico (suelo, clima, disponibilidad de agua, etc.) y factores políticos y socioeconómicos, que las teorías clásicas y de las nuevas de geografía económica privilegian. Bajo estas circunstancias, parece apropiado dar especial atención a la lógica y dinámica de los agentes del capital global, que es el principal responsable de los cambios intensificados y acelerados sobre la geografía de la producción. Esto es, el capital global que cada vez más se concentra en enormes corporaciones transnacionales que adoptan diversas estrategias de producción, desde niveles muy altos de integración vertical a través de compras y fusiones, hasta un gran uso de esquemas de subcontratación con capitalistas locales, cooperativas y productores menores de mercancías. Estas estrategias incrementan el impacto de los efectos de escala, especialmente a aquellas producciones agrícolas mas sensibles a la escala, tales como la ganadería intensiva o ganadería de pastoreo. Esto significa que la mayor parte de la producción masiva, se concentra cada día más en aquellas áreas mas adecuadas para esos propósitos. Esto es, la producción de alimentos se concentra en áreas más favorables (Belo-Moreira, 2004).

Por otra parte, las Fundaciones y ONG's han mostrado sobrado interés en apoyar a las organizaciones campesinas, se destaca: i) El hecho de tener una imagen más humana, más sensible que les permita cierta legitimación ante la sociedad, muchas empresas trasnacionales en función de ser altamente eficiente y productivas, han hecho un uso indiscriminado de los recursos naturales, han subordinado la actividad agropecuarias a sus intereses, han degradado el medio ambiente ocasionado daños irreversibles, tales como el calentamiento global, degradación de suelos, etc,. Esto ha creado una imagen negativa de estas grandes corporaciones y ii) La desarticulación que existe entre estos grupos corporativos y los gobiernos, estos grupos corporativos debido a la gran cantidad de recursos que manejan, operan de manera desmedida, ellos saben que ciertas acciones se pueden revertir en un futuro, para contrarrestar ciertas amenazas futuras, las empresas requieren de una estructura legal que los proteja, pero no todos los gobiernos acceden a dicha pretensión, y una manera coercitiva, es desviando los ingresos de dichos gobiernos por conceptos de ingresos para aquello gobiernos que tienen alta dependencia de estos recursos.

\section{La innovación tecnológica}

La tecnología es un recurso que adquiere cuerpo no sólo en el capital físico, sino también en las habilidades humanas y en las instituciones y estructuras sociales. La 
tecnología no es un recurso dado y estático, sino un conjunto de capacidades dinámicas utilizadas para absorber, adaptar y avanzar los conocimientos y habilidades productivas existentes (Villazul, 1999). Otra definición de tecnología la señala Freeman (1974. Citado por Villazul, 1999), donde destaca que la tecnología es un conjunto de conocimientos acerca de técnicas que pueden abarcar tanto el conocimiento en sí como la materialización tangible de ese conocimiento en un proceso productivo, en un sistema operativo o en la maquinaria y el equipo físico de producción. Esta definición incluye la "tecnología no incorporada" como el conocimiento y las técnicas, así como la "tecnología incorporada" en un sistema operativo físico o intangible.

La innovación tecnológica es la transformación de una idea, ya sea en un producto nuevo o ya sea mejorado que se introduce en el mercado (innovación de producto), ya sea en un proceso operacional que se adopte en la industria y en el comercio (innovación de proceso) (Villazul, 1999). Freeman (1974. Citado por Villazul, 1999), destaca que la innovación tecnológica abarca los avances en el conocimiento y en la introducción y difusión de productos y procesos nuevos, mejorados en la economía. Una innovación sólo tiene lugar cuando se produce la primera transacción comercial en la que interviene ese producto, proceso, sistema o dispositivo nuevos, si bien el término se utiliza para designar al proceso completo. Este proceso va estar vinculado a actividades de adaptación, asimilación y aprendizaje de habilidades y conocimientos. En función de este planteamiento teórico podemos concluir que la innovación tecnológica es un proceso dinámico, que se genera a partir de un factor que es la adaptación, este a su vez a través de la dialéctica hombre-naturaleza genera habilidades y conocimientos que se acumulan y retroalimentar en tiempo y espacio. En un ambiente que se esta haciendo cada vez más orientado al mercado, y en el que hay una tendencia a rechazar agricultores de bajos recursos, constituye una preocupación critica encontrar salidas favorables a este fenómeno, y es a partir de aquí que el conocimiento local y/o indígena toma una importancia estratégica.

\section{El conocimiento local}

En las condiciones actuales del entorno global, regional y local, el conocimiento adquiere su máxima relevancia. El esfuerzo que deben realizar las naciones en este sentido se efectúa en diversas escalas: por ejemplo, aspectos tales como la definición de políticas de conocimiento; el incremento de las inversiones en investigación y desarrollo, en educación e innovación; así como, la articulación de las acciones dispersas ejecutadas por centros productores de conocimientos, las empresas y el sector público corresponden a la escala nacional. Los esfuerzos por impulsar la innovación tecnológica, la generación y aplicación de conocimientos en la búsqueda de una producción ambientalmente sostenible y la formación y capacitación de la fuerza laboral, las comunidades y las familias rurales, adquiere su mayor eficacia en la escala local (Alfaro, 2003).

El surgimiento del tema del conocimiento local como parte importante en las agendas de desarrollo de las entidades multilaterales es a su vez parte de la construcción de un paradigma novedoso que encuentra en los conocimientos de las culturas del mundo una oportunidad para articular sus propias políticas de desarrollo. El conocimiento local va estar vinculado a la cultura (identidad, espiritualidad y creencias) y el mismo se proyecta como un medio para resolver ciertos problemas de desarrollo, desde la visión

Revista Mad. N 16, Mayo de 2007. Departamento de Antropología. Universidad de Chile 
indígena es un apéndice importante de la cultura, vital para la supervivencia global (Perafán, 2004 y Kolawole, 2001).

Numerosos estudiosos interesados en el conocimiento local subrayan la importancia de aprender de personas cuya visión del mundo y prácticas han sido moldeadas por un vínculo íntimo con su territorio biofísico, sumamente específico y único (Li, 2002). Sería correcto puntualizar que el actual interés por el conocimiento indígena es fruto en buena medida de la general aceptación del vínculo establecido entre ese conocimiento y el desarrollo.

El rescate de un saber ancestral a menudo revaloriza bienes y servicios muy elementales, pero que son valorados precisamente por su carácter limpio y orgánico (en el caso de productos agrícolas) o por su carácter sui generis al ser el resultado de prácticas productivas profundamente incrustadas en algunas culturas, como es el caso de muchos productos artesanales. Saber "codificado" y saber "tácito" se dan la mano a través de nuevos o renovados productos (Boissier, 2001).

El conocimiento local es un fenómeno que surge y se desarrolla a través de prácticas históricamente localizadas nacido de una experiencia arraigada., en contextos sociales e institucionales específicos. (Agrawal, 1995; Murdoch y Clark, 1994 citado por Leach y Fairhead, 2002; Sundar, 2002). Se desarrolla en relación con ámbitos específicos del poder. Además, es recursivo, es decir, está sujeto a bucles de retroalimentación mediante los cuales un grupo aprende de otro e integra nuevas maneras de pensar (Li, 2002).

Las comunidades campesinas e indígenas mantienen un diálogo constante con otra gente y otros lugares, reafirmando y reinventando constantemente su propio carácter local en relación con el sinnúmero de otros lugares en los que participan física, imaginativa y culturalmente, y a través de las redes en expansión de la economía política y cultural translocal. Como todos nosotros, están aprendiendo constantemente cosas nuevas acerca del mundo en que viven: su gente, sus ríos, sus plantas, sus suelos. Están hablando, escuchando e intercambiando ideas constantemente acerca de las cosas importantes para ellos, estableciendo conexiones en el tiempo y el espacio, a través de la radio, la televisión y los vídeos, a través de agentes de promoción social, sindicalistas, especialistas gubernamentales e investigadores extranjeros, y a través de todo tipo de mercancías ambulantes (Raffles, 2002).

\section{Conclusión}

Este breve análisis de los elementos mencionados anteriormente es fundamental para sentar las bases de un desarrollo territorial rural, donde no solo se considera el espacio rural-urbano, sino que su interacción se precisa para el logro de tal objetivo. Un aspecto de relevancia y que fue poco discutido; es la dinámica del medio ambiente y el papel que juegan las organizaciones campesinas. En particular, el papel del sector público se esta reduciendo y se esta poniendo nuevo énfasis en crear espacio para el sector privado y en desarrollar relaciones institucionales para la provisión de servicios y la gestión de recursos naturales. RM 


\section{Bibliografía}

AGRAWAL, A. 2002. "El conocimiento indígena y la dimensión política de la clasificación". Revista Internacional de Ciencias Sociales. 173:6-18.

ALFARO, J. 2003. "Gestión del Conocimiento y el desarrollo rural en América Latina y el Caribe". Seminario: La reconstrucción de las instituciones rurales en el ámbito de los servicios de asistencia técnica. Antigua: FODEPAL-AECI-I NIA.

ÁVILA, H. 1999. "La dinámica actual de los territorios rurales en América Latina". Scripta Nova. 45 (40). Disponible En: www.ub.es/geocrit/sn-4540.htm.

BARTRA, A. 1998. "Globalización, crisis, y desarrollo rural en América Latina". Asociación Latinoamericana de Sociología Rural (ALASRU). V Congreso Latinoamericano de Sociología Rural. Mexico, D.F: Memorias.

BELO-MOREIRA, M. 2004. "La nueva territorializacion del espacio rural en el contexto de la globalización: El espíritu emprendedor en las áreas marginales". Rev. Agricultura, Sociedad y Desarrollo. 1:85-100.

BOISIER, S. 2001. Sociedad del conocimiento, conocimiento social y gestión territorial. Santiago: Pontificia Universidad Católica de Chile.

CALVILLO, M. y FAVELA. A. 1995. “Los nuevos sujetos sociales. Una aproximación epistemológica". Rev. Sociológica. 28:251-278.

KOLAWOLE, O. D. 2001. "Local Knowledge utilization and sustainable rural development in the $21^{\text {st }}$ century". Indigenous Knowledge and Development Monitor. 9 (3): 13-15. Disponible En: www.nuffic.nl/ik-pages/index.html.

LEACH, M y J. FAIRHEAD. 2002. "Modo de Contestación: la "ciencia ciudadana" y los "conocimientos indígenas" en África occidental y el Caribe". Revista Internacional de Ciencias Sociales. 173: 18-35.

LI, T. M. 2002. "La limpieza étnica, los conocimientos recursivos y los dilemas del sedentarismo". Revista Internacional de Ciencias Sociales. 173: 94-107.

LUGO, D. 2005. “Reflexión teórica sobre la validez de los preceptos de acción social de Max weber para el análisis del campesinado actual". Revista Ambiente $\&$ Sociedade. 8 (2): 1-9.

LUSTIG, N. C y Székely. M. 1997. Mexico: Evolución económica, pobreza y desigualdad. Documento de trabajo. Washington D.C: PNUD-BID-CEPAL.

PERAFÁN, C. 2004. Análisis de usos culturales de la tierra: El concepto de uso cultural de la tierra. Documento de trabajo. Washington, D.C: CLAN-BID-EPFL.

RAFFLES, H. 2002. "El conocimiento intimo". Revista Internacional de Ciencias Sociales. 173:49-61. 
RAMÍREZ, J y PEÑA. B. 1999. “Agricultura campesina e industrialización en el valle de puebla". Ponencia preparada para el Congreso Nacional sobre Políticas de Ajuste Estructural en el Campo Mexicano: Efectos y Respuestas. Disponible En: www.unam.mx/rer/AjusteP.html.

SCHEJ TMAN, A y BERDEGUÉ. J. 2003. Desarrollo Territorial Rural. Documento de Trabajo. División América Latina y el Caribe del Fondo Internacional de Desarrollo Agrícola y el Departamento de Desarrollo Sustentable del Banco Interamericano de Desarrollo. RIMISP. Santiago de Chile.

SILVA, I. 2003. Metodología para la elaboración de estrategias de desarrollo local. Instituto Latinoamericano y del Caribe de Planificación Económica y Social (ILPES/CEPAL). Documento de trabajo № 42. Santiago de Chile.

SUNDAR, N. 2002. "Indigenizar, nacionalizar y espiritualizar-¿un programa para la educación?". Revista Internacional de Ciencias Sociales. 173:49-61.

THORNER, D. 1979. La economía campesina. Concepto para la historia económica. En: Economía Campesina, Centro de Estudios y Promoción del Desarrollo (DESCO). Lima: 139-153 pp.

VILLAZUL, J. J. 1999. Los sistemas de innovación como mecanismos de innovación y de transferencia tecnológica. Documento de Trabajo № 76. México, D.F: Centro de Investigación y Docencia Económicas.

WARMAN, A. 2001. El campo mexicano en el siglo XX. México, D.F: Fondo de Cultura Económica. 280 p.

\section{Notas}

\footnotetext{
${ }^{1}$ La categoría de sujeto social remite al terreno en donde se construyen las subjetividades (entendiéndose por subjetividad la particular concepción del mundo y de la vida del sujeto) colectivas, las identidades y la voluntad de mutación (entendiéndose por voluntad de mutación a lo verdaderamente esperanzado en el sujeto, a la disposición al riesgo y al triunfo de lo querido utópicamente) (Calvillo y Favela, 1995).
} 